TRANSACTIONS OF THE

AMERICAN MATHEMATICAL SOCIETY

Volume 352, Number 11, Pages 5063-5076

S 0002-9947(00)02637-4

Article electronically published on June 28, 2000

\title{
EUCLIDEAN WEIGHTS OF CODES FROM ELLIPTIC CURVES OVER RINGS
}

\author{
JOSÉ FELIPE VOLOCH AND JUDY L. WALKER
}

\begin{abstract}
We construct certain error-correcting codes over finite rings and estimate their parameters. For this purpose, we need to develop some tools, notably an estimate for certain exponential sums and some results on canonical lifts of elliptic curves. These results may be of independent interest.
\end{abstract}

\section{INTRODUCTION}

A code is a subset of $A^{n}$, where $A$ is a finite set (called the alphabet). Usually $A$ is just the field of two elements and, in this case, one speaks of binary codes. Such codes are used in applications where one transmits information through noisy channels. By building redundancy into the code, transmitted messages can be recovered at the receiving end. A code has parameters that measure its efficiency and error-correcting capability. For various reasons one often restricts attention to linear codes, which are linear subspaces of $A^{n}$ when $A$ is a field. However, there are non-linear binary codes (such as the Nordstrom-Robinson, Kerdock, and Preparata codes) that outperform linear codes for certain parameters. These codes have remained somewhat mysterious until recently when Hammons, et al. ([] $]$ ) discovered that one can obtain these codes from linear codes over rings (i.e. submodules of $A^{n}, A$ a ring) via the Gray mapping, which we recall below.

In a different vein, over the last decade there has been a lot of interest in linear codes coming from algebraic curves over finite fields. The construction of such codes was first proposed by Goppa in [5]; see [15] or [16] for instance. In [17, it is proven that for $q \geq 49$ a square, there exist sequences of codes over the finite field with $q$ elements which give asymptotically the best known linear codes over these fields. The second author has extended Goppa's construction to curves over local Artinian rings and shown, for instance, that the Nordstrom-Robinson code can be obtained from her construction followed by the Gray mapping; see [20] and 21]. While most of the parameters for these new codes were estimated in the above papers, the crucial parameter needed to describe the error-correcting capability of the images of these codes under the Gray mapping was still lacking. In this paper we consider the second author's construction in the special case of elliptic curves which are defined over finite local rings and which are the canonical lifts of their reductions. (See section 4 for more about canonical lifts.) For these codes, the missing parameter can be estimated, and we do so.

Received by the editors October 29, 1998 and, in revised form, September 16, 1999.

1991 Mathematics Subject Classification. Primary 94B27; Secondary 11T71, 11 G07.

The first author was supported in part by NSA Grant \#MDA904-97-1-0037.

The second author was supported in part by NSF Grant \#DMS-9709388. 
Another application of our construction is to obtain low-correlation sequences suitable for use in code-division multiple access (CDMA) schemes, which are used when multiple users need to share a common communication channel, such as in the case of cellular telephones. We will use our results to obtain such sequences. In a way, our results are the analogues for elliptic curves of the results of Kumar et al. (8]), which can be viewed as being for the multiplicative group. Since we can work with any ordinary elliptic curve over a finite field, our results are more flexible. This flexibility should eventually lead to more examples of good codes; see section 6 for further discussion on this.

This paper is organized as follows. In section 2 we recall the main results of 20] on the construction of codes from curves over rings, and review the definitions pertaining to error-correcting codes. We also set the stage in this section for the results we need. In section 3 we prove a general estimate for certain exponential sums along curves. This result extends a number of recent results but, paradoxically, is based on an old paper of H. L. Schmid. In section 4 we prove a number of results about canonical lifts of elliptic curves. In section 5, we put everything together, obtaining our main results and their applications. Finally, in section 6 , we look at the problem of constructing examples using our methods.

\section{Algebraic GeOMEtric CODES OVER RINGS}

In 20], the idea of algebraic geometric codes over rings other than fields is introduced, and foundational results about these codes are proven. In [21], the methods of [20] are used to explicitly construct the $\mathbb{Z} / 4 \mathbb{Z}$-version of the NordstromRobinson code as an algebraic geometric code. In order to construct other codes over $\mathbb{Z} / 4 \mathbb{Z}$ with good nonlinear binary shadows, we must first investigate the Lee and Euclidean weights of these codes. In this section, we recall the definitions and some results from [20] and explain how the Lee and Euclidean weights of algebraic geometric codes over rings are related to exponential sums.

Let $A$ be a local Artinian ring with maximal ideal $\mathfrak{m}$. We assume that the field $A / \mathfrak{m}$ is finite; say $A / \mathfrak{m}=\mathbb{F}_{q}$. Let $\mathbf{X}$ be a curve over $A$, that is, a connected irreducible scheme over $\operatorname{Spec} A$ which is smooth of relative dimension one. Let $\mathbf{X} \times_{\operatorname{Spec} A} \operatorname{Spec} \mathbb{F}_{q}=X \subset \mathbf{X}$ be the fiber of $\mathbf{X}$ over the closed point of $\operatorname{Spec} A$. We assume $X$ is absolutely irreducible, so that it is the type of curve on which algebraic geometric codes over $\mathbb{F}_{q}$ are defined. Let $\mathcal{Z}=\left\{Z_{1}, \ldots, Z_{n}\right\}$ be a set of $A$-points on $\mathbf{X}$ with distinct specializations $P_{1}, \ldots, P_{n}$ in $X$. Let $G$ be a (Cartier) divisor on $\mathbf{X}$ such that no $P_{i}$ is in the support of $G$, and let $\mathcal{L}=\mathcal{O}_{\mathbf{X}}(G)$ be the corresponding line bundle. For each $i$, we have $\Gamma\left(Z_{i},\left.\mathcal{L}\right|_{Z_{i}}\right) \simeq A$, and thinking of elements of $\Gamma(\mathbf{X}, \mathcal{L})$ as rational functions on $\mathbf{X}$, we may think of the composition $\Gamma(\mathbf{X}, \mathcal{L}) \rightarrow$ $\Gamma\left(Z_{i},\left.\mathcal{L}\right|_{Z_{i}}\right) \rightarrow A$ as evaluation of these functions at $Z_{i}$. Summing over all $i$, we have a map $\gamma: \Gamma(\mathbf{X}, \mathcal{L}) \rightarrow \bigoplus \Gamma\left(Z_{i},\left.\mathcal{L}\right|_{Z_{i}}\right) \rightarrow A^{n}$, given by $f \mapsto\left(f\left(Z_{1}\right), \ldots, f\left(Z_{n}\right)\right)$.

Definition 2.1. Let $A, \mathbf{X}, \mathcal{Z}, \mathcal{L}$, and $\gamma$ be as above. Define $C_{A}(\mathbf{X}, \mathcal{Z}, \mathcal{L})$ to be the image of $\gamma \cdot C_{A}(\mathbf{X}, \mathcal{Z}, \mathcal{L})$ is called the algebraic geometric code over $A$ associated to $\mathbf{X}, \mathcal{Z}$, and $\mathcal{L}$.

The following theorem summarizes some of the main results of [20].

Theorem 2.2. Let $A, \mathbf{X}, \mathcal{L}$, and $\mathcal{Z}=\left\{Z_{1}, \ldots, Z_{n}\right\}$ be as above. Let $g$ denote the genus of $\mathbf{X}$, and suppose $2 g-2<\operatorname{deg} \mathcal{L}<n$. Set $C=C(\mathbf{X}, \mathcal{Z}, \mathcal{L})$. Then $C$ is a linear code of length $n$ over $A$, and is free as an A-module. The dimension (rank) 
of $C$ is $k=\operatorname{deg} \mathcal{L}+1-g$, and the minimum Hamming distance of $C$ is at least $n-\operatorname{deg} \mathcal{L}$. Further, under the additional assumption that $A$ is Gorenstein, the class of algebraic geometric codes is closed under taking duals. In particular, there exists a line bundle $\mathcal{E}$ such that $C^{\perp}=C(\mathbf{X}, \mathcal{Z}, \mathcal{E})$.

Remark 2.3. The minimum Hamming distance is obtained by comparing zeros and poles, and the dimension computation is a consequence of the Riemann-Roch Theorem. These estimates require the assumption $2 g-2<\operatorname{deg} \mathcal{L}<n$. The duality result follows from a generalized version of the Residue Theorem which holds for Gorenstein rings. See [20] for details.

The following result will be useful in section 5 .

Lemma 2.4. Let $A, \mathbf{X}$, and $\mathcal{L}$ be as in Theorem 2.2 and let $p$ be the characteristic of the residue field of $A$. Let $\mathbf{K}$ be the total quotient ring of rational functions on $\mathbf{X}$, and let $\mathbf{L}=\Gamma(\mathbf{X}, \mathcal{L})$. Define $p^{-1} \mathbf{L}=\{\mathbf{g} \in \mathbf{K} \mid p \mathbf{g} \in \mathbf{L}\}$. Then $p^{-1} \mathbf{L}=\mathbf{L}+\operatorname{ker}(\beta)$, where $\beta$ is the map $\mathbf{K} \rightarrow \mathbf{K}$ given by multiplication by $p$.

Proof. Consider the map of the short exact sequence $0 \rightarrow \mathbf{L} \rightarrow \mathbf{K} \rightarrow \mathbf{K} / \mathbf{L} \rightarrow 0$ to itself given by multiplication by $p$. By the Snake Lemma, the kernels and cokernels fit into an exact sequence. But it is shown in [20] that $\mathbf{L} \otimes_{A} A / m=\Gamma\left(X, \mathcal{L}^{\prime}\right)$, where $\mathcal{L}^{\prime}$ is the pullback of $\mathcal{L}$ to $X$. This means that the cokernels form a short exact sequence by themselves, so the kernels must also. To set up notation, let $\gamma$ : $\mathbf{K} / \mathbf{L} \rightarrow \mathbf{K} / \mathbf{L}$ be multiplication by $p$, let $\pi_{0}$ be the surjection $\operatorname{ker}(\beta) \rightarrow \operatorname{ker}(\gamma)$, and let $\pi$ be the surjection $\mathbf{K} \rightarrow \mathbf{K} / \mathbf{L}$. With this notation, $p^{-1} \mathbf{L}=\operatorname{ker}(\pi \beta)=\operatorname{ker}(\gamma \pi)$.

Let $\mathbf{g} \in p^{-1} \mathbf{L}$. Then $\pi(\mathbf{g}) \in \operatorname{ker}(\gamma)$. Since $\pi_{0}$ is surjective, there is some $\mathbf{g}^{\prime} \in$ $\operatorname{ker}(\beta)$ with $\pi_{0}\left(\mathbf{g}^{\prime}\right)=\pi(\mathbf{g})$. But then $\pi\left(\mathbf{g}-\mathbf{g}^{\prime}\right)=0$, so $\mathbf{g}-\mathbf{g}^{\prime} \in \mathbf{L}$. In other words, there is some $\mathbf{f}^{\prime} \in \mathbf{L}$ with $\mathbf{g}=\mathbf{f}^{\prime}+\mathbf{g}^{\prime}$, which is precisely what we needed to show.

For applications, one is usually concerned with constructing codes over $\mathbb{Z} / 4 \mathbb{Z}$, or more generally, over rings of the form $\mathbb{Z} / p^{l} \mathbb{Z}$, where $p$ is prime and $l \geq 1$. We can use algebraic geometry to construct such codes in two different ways. First, we can simply set $A=\mathbb{Z} / p^{l} \mathbb{Z}$ in the definition of algebraic geometric codes above. Alternatively, we can construct an algebraic geometric code over $G R\left(p^{l}, m\right)$ and look at the associated trace code over $\mathbb{Z} / p^{l} \mathbb{Z}$. Here, $G R\left(p^{l}, m\right)$ denotes the degree $m \geq 1$ Galois extension of $\mathbb{Z} / p^{l} \mathbb{Z}$ (see, for example, [8] for details). It is easily seen that such a ring is isomorphic to the ring of length $l$ Witt vectors over the field $\mathbb{F}_{p^{m}}$, and this representation is used in sections 3 and 4 below. In particular, there is a trace map $T: G R\left(p^{l}, m\right) \rightarrow \mathbb{Z} / p^{l} \mathbb{Z}$, and by the trace code of a code, we mean the code obtained by applying this trace map coordinatewise to the codewords.

The Gray map allows us to construct (non-linear) binary codes from codes over $\mathbb{Z} / 4 \mathbb{Z}$, and is defined as follows. Consider the map $\varphi: \mathbb{Z} / 4 \mathbb{Z} \rightarrow \mathbb{F}_{2}^{2}$ defined by $\varphi(0)=(0,0), \varphi(1)=(0,1), \varphi(2)=(1,1), \varphi(3)=(1,0)$. Now we define a map, again denoted by $\varphi:(\mathbb{Z} / 4 \mathbb{Z})^{n} \rightarrow \mathbb{F}_{2}^{2 n}$, by applying the previous $\varphi$ to each coordinate.

For linear codes over rings of the form $\mathbb{Z} / p^{l} \mathbb{Z}$, it is often either the Euclidean or Lee weight rather than the Hamming weight which is of interest. In particular, when $p^{l}=4$, the Euclidean and Lee weights are closely related, and the Lee weight gives the Hamming weight of the associated nonlinear binary code. 
We begin by defining Euclidean weights. We identify an element $x$ of the cyclic group $\mathbb{Z} / p^{l} \mathbb{Z}$ with the corresponding $p^{l}$ th root of unity via the map

$$
x \rightarrow e_{p^{l}}(x):=e^{2 \pi i x / p^{l}} .
$$

Definition 2.5. The Euclidean distance between $x$ and $y$ is the distance $d_{E}(x, y)$ in the complex plane between the points $e_{p^{l}}(x)$ and $e_{p^{l}}(y)$, and the Euclidean weight of $x$ is the distance $w_{E}(x)$ between $e_{p^{l}}(x)$ and $e_{p^{l}}(0)=1$.

We have

$$
w_{E}(x)=\sqrt{\sin ^{2}\left(\frac{2 \pi x}{p^{l}}\right)+\left(1-\cos \left(\frac{2 \pi x}{p^{l}}\right)\right)^{2}}=\sqrt{2-2 \cos \left(\frac{2 \pi x}{p^{l}}\right)} .
$$

In fact, it is usually the square of the Euclidean weight in which one is interested. This is given by $w_{E}^{2}(x)=2-2 \cos \left(\frac{2 \pi x}{p^{L}}\right)$. For vectors $\mathbf{x}=\left(x_{1}, \ldots, x_{n}\right)$ and $\mathbf{y}=$ $\left(y_{1}, \ldots, y_{n}\right)$ over $\mathbb{Z} / p^{l} \mathbb{Z}$, we define

$$
d_{E}^{2}(\mathbf{x}, \mathbf{y})=\sum_{j=1}^{n} d_{E}^{2}\left(x_{j}, y_{j}\right)
$$

and

$$
w_{E}^{2}(\mathbf{x})=\sum_{j=1}^{n} w_{E}^{2}\left(x_{j}\right) .
$$

For example, the squared Euclidean weight of the all-one vector in $\left(\mathbb{Z} / p^{l}\right)^{n}$ is $2 n\left(1-\cos \left(2 \pi / p^{l}\right)\right)$. Using the Taylor expansion of cosine, we get that this is at least $4 n \frac{\pi^{2}}{p^{2 l}}\left(1+\frac{\pi^{2}}{3 p^{2 l}}\right)$. Further, any other nonzero multiple of the all-one vector in $\left(\mathbb{Z} / p^{l}\right)^{n}$ has squared Euclidean weight at least this.

For general vectors, since $\cos \left(\frac{2 \pi x}{p^{l}}\right)=\operatorname{Re}\left(e_{p^{l}}(x)\right)$, we have

$$
\begin{aligned}
w_{E}^{2}(\mathbf{x}) & =\sum_{j=1}^{n}\left(2-2 \operatorname{Re}\left(e_{p^{l}}\left(x_{j}\right)\right)\right) \\
& =2 n-2 \operatorname{Re} \sum_{j=1}^{n} e_{p^{l}}\left(x_{j}\right) \\
& \geq 2 n-2\left|\sum_{j=1}^{n} e_{p^{l}}\left(x_{j}\right)\right| .
\end{aligned}
$$

Hence, to find a lower bound on the minimum Euclidean weight of a linear code over $\mathbb{Z} / p^{l} \mathbb{Z}$, it is enough to find an upper bound on the modulus of the exponential sum

$$
\sum_{j=1}^{n} e_{p^{l}}\left(x_{j}\right)
$$

Now consider the case $p^{l}=4$. Then $e_{4}(0)=1, e_{4}(1)=i, e_{4}(2)=-1$, and $e_{4}(3)=-i$. Hence $w_{E}^{2}(0)=0, w_{E}^{2}(1)=w_{E}^{2}(3)=2$, and $w_{E}^{2}(2)=4$. Since the Lee weight is defined by $w_{L}(0)=0, w_{L}(1)=w_{L}(3)=1$, and $w_{L}(2)=2$, we have

$$
w_{L}(x)=\frac{1}{2} w_{E}^{2}(x)
$$


for any $x \in \mathbb{Z} / 4 \mathbb{Z}$. From this we see that the Euclidean weight of a codeword over $\mathbb{Z} / 4 \mathbb{Z}$ is twice the Hamming weight of the binary codeword obtained by applying the Gray map. Notice that the Lee weight of any multiple of the all-one vector in $(\mathbb{Z} / 4 \mathbb{Z})^{n}$ is either $0, n$, or $2 n$.

Finally, let $C$ be an algebraic geometric code over $G R\left(p^{l}, m\right)$, and let $T$ : $G R\left(p^{l}, m\right) \rightarrow \mathbb{Z} / p^{l} \mathbb{Z}$ denote the trace map as before. We are interested in the minimum Euclidean weight of $T(C)$, the trace code of $C$, which is a linear code over $\mathbb{Z} / p^{l} \mathbb{Z}$. Codewords in $T(C)$ are of the form $\left(T\left(f\left(Z_{1}\right)\right), \ldots, T\left(f\left(Z_{n}\right)\right)\right)$, where $f$ is a rational function on some curve $\mathbf{X}$ defined over $G R\left(p^{l}, m\right)$ and $Z_{1}, \ldots, Z_{n}$ are $G R\left(p^{l}, m\right)$-points on $\mathbf{X}$. From the argument above, to find a lower bound for the minimum Euclidean weight of $T(C)$ it suffices to find an upper bound on the modulus of

$$
\sum_{j=1}^{n} e_{p^{l}}\left(T\left(f\left(Z_{j}\right)\right)\right)=\sum_{j=1}^{n} e^{2 \pi i T\left(f\left(Z_{j}\right)\right) / p^{l}} .
$$

We investigate this sum in sections 3 and 4 below.

\section{EXPONENTIAL SUMS}

In this section we will give estimates for some kinds of exponential sums along curves. The approach follows the classical method of relating the exponential sum to the sum of the reciprocals of the zeros of an $L$-function and applying the Riemann hypothesis. Of course the abstract set-up is well-known in even greater generality (see, for example, [4]), but it is the calculation of the degree of the $L$-function that requires working out. We will be dealing with characters of order $p^{l}$, where $p$ is the characteristic. In this case, the degree of the $L$-function was computed by Schmid [13], 14].

Let $X$ be a curve over the finite field $\mathbb{F}_{q}$, where $q=p^{m}$ with $p$ prime. Denote by $K=\mathbb{F}_{q}(X)$ the function field of $X$. Let $f_{0}, \ldots, f_{l-1} \in K$ and consider the Witt vector $\mathbf{f}=\left(f_{0}, \ldots, f_{l-1}\right) \in W_{l}(K)$. Let $X_{0}$ be the maximal affine open subvariety of $X$ where $f_{0}, \ldots, f_{l-1}$ do not have poles, and let $P \in X_{0}\left(\mathbb{F}_{q}\right)$. We can then consider the Witt vector $\mathbf{f}(P)=\left(f_{0}(P), \ldots, f_{l-1}(P)\right) \in W_{l}\left(\mathbb{F}_{q}\right)$. Letting $T: W_{l}\left(\mathbb{F}_{q}\right) \rightarrow W_{l}\left(\mathbb{F}_{p}\right) \cong \mathbb{Z} / p^{l} \mathbb{Z}$ denote the trace map as in section 2, we can consider the exponential sum

$$
S_{\mathbf{f}, \mathbb{F}_{q}}=\sum_{P \in X_{0}\left(\mathbb{F}_{q}\right)} e^{2 \pi i T(\mathbf{f}(P)) / p^{l}}
$$

Theorem 3.1. With notation as above, assume $X \backslash X_{0}$ consists of the points above the valuations $v_{1}, \ldots, v_{s}$ of $K$. Let $g$ be the genus of $X$, and for $i=0, \ldots, l-1$, $j=1, \ldots, s$, define $n_{i j}=-v_{j}\left(f_{i}\right)$. Assume that $\mathbf{f}$ is not of the form $\mathbf{f}=F(\mathbf{g})-\mathbf{g}+\mathbf{c}$ for any $\mathbf{g} \in W_{l}(K)$ and $\mathbf{c} \in W_{l}\left(\mathbb{F}_{q}\right)$, where $F$ denotes the additive endomorphism on $W_{l}(K)$ given by $F\left(g_{0}, g_{1}, \ldots, g_{l-1}\right)=\left(g_{0}^{p}, g_{1}^{p}, \ldots, g_{l-1}^{p}\right)$. Then $\left|S_{\mathbf{f}, \mathbb{F}_{q}}\right| \leq B q^{1 / 2}$, where

$$
B \leq 2 g-1+\sum_{j=1}^{s} \max \left\{p^{l-1-i} n_{i j} \mid 0 \leq i \leq l-1\right\} \operatorname{deg} v_{j} .
$$

Proof. As mentioned above, the essential steps are done in [13], 14], but we will repeat them here for the reader's convenience. Consider the Artin-Schreier-Witt extension $Y: F(\mathbf{y})-\mathbf{y}=\mathbf{f}$ of $X$, which is a cover of $X$ with Galois group contained 
in $\mathbb{Z} / p^{l} \mathbb{Z}$. Assume first it is a geometric cover, i.e., that there is no constant field extension, and that it has positive degree. If $\chi$ is a character of the Galois group then we can form the (Artin) $L$-function $L(X, \chi, t)$. As long as $\chi \neq 1, L(X, \chi, t)$ is a polynomial in $t$ of a certain degree $B_{\chi}$ satisfying

$$
B_{\chi} \leq 2 g-1+\sum_{j=1}^{s} \max \left\{p^{l-1-i} n_{i j} \mid 0 \leq i \leq l-1\right\} \operatorname{deg} v_{j},
$$

with equality holding if $\chi$ is injective (see [13, [14], and especially [14, Satz 8). When $\chi=1, L(X, \chi, t)$ is the zeta function of $X$. Taking the product over all characters $\chi$ of the Galois group of $Y / X$, the function $\prod L(X, \chi, t)$ is the zeta function of $Y$. From this our bound will follow since, by the general theory (e.g. 14] (2.7)), $S_{\mathbf{f}, \mathbb{F}_{q}}=\sum_{P \in X_{0}\left(\mathbb{F}_{q}\right)} \chi(P)$ for a character $\chi$, where $\chi(P)$ means $\chi$ evaluated at the Frobenius substitution of $P$. Also, $\sum_{P \in X_{0}\left(\mathbb{F}_{q}\right)} \chi(P)$ equals the negative of the sum of the reciprocals of the roots of $L(X, \chi, t)$, and these roots have absolute value $q^{-1 / 2}$ by the Riemann hypothesis.

We now treat the case where $Y$ is not necessarily a geometric cover of $X$. Let $L=K(\mathbf{y})$, so $L / K$ is cyclic. Now if $\mathbb{F}_{q}$ is not algebraically closed in $L$, then $L$ contains $k=\mathbb{F}_{q^{p}}$. Set $M=K k \subset L$, so that $[M: K]=\left[k: \mathbb{F}_{q}\right]=p$. Since $L$ is cyclic, the intermediate field extension of degree $p$ over $K$ is unique, so we have $M=K\left(y_{0}\right)$. Thus $K\left(y_{0}\right) / K$ is a constant field extension, which implies that $f_{0}=g^{p}-g+a$ for some $g \in K$ and $a \in \mathbb{F}_{q}$. Letting $\mathbf{g}=(g, 0, \ldots, 0) \in W_{l}(K)$ and $\mathbf{a}=(a, 0, \ldots, 0) \in W_{l}\left(\mathbb{F}_{q}\right)$, we can find $\mathbf{h} \in W_{l-1}(K)$ such that $\mathbf{f}=F(\mathbf{g})-\mathbf{g}+\mathbf{a}+p \mathbf{h}$. Then

$$
S_{\mathbf{f}, \mathbb{F}_{q}}=e^{2 \pi i T(\mathbf{a})} \sum_{P \in X_{0}\left(\mathbb{F}_{q}\right)} e^{2 \pi i T(\mathbf{h}(P)) / p^{l-1}} .
$$

Notice that if $\mathbf{h}=F(\mathbf{k})-\mathbf{k}+\mathbf{d}$ with $\mathbf{k} \in W_{l-1}(K)$ and $\mathbf{d} \in W_{l-1}\left(\mathbb{F}_{q}\right)$, then $\mathbf{f}=F(\mathbf{g})-\mathbf{g}+\mathbf{a}+p(F(\mathbf{k})-\mathbf{k}+\mathbf{d})=F(\mathbf{g}+p \mathbf{k})-(\mathbf{g}+p \mathbf{k})+(\mathbf{a}+\mathbf{d})$, which contradicts the hypothesis of the theorem. Therefore, we can assume by induction on $l$ that $\left|S_{\mathbf{f}, \mathbb{F}_{q}}\right| \leq C q^{1 / 2}$, with

$$
C \leq 2 g-1+\sum_{j=1}^{s} \max \left\{p^{l-2-i} m_{i j} \mid 0 \leq i \leq l-2\right\} \operatorname{deg} v_{j},
$$

where $m_{i j}=-v_{j}\left(h_{i}\right)$. Further, a computation gives $m_{i} \leq \max \left\{p^{i-j} n_{j} \mid 0 \leq j \leq\right.$ $i+1\}$, so in fact

$$
C \leq 2 g-1+\sum_{j=1}^{s} \max \left\{p^{l-2-i} n_{i j} \mid 0 \leq i \leq l-1\right\} \operatorname{deg} v_{j},
$$

and from this the theorem follows.

Remark 3.2. There has been some recent interest in exponential sums of the kind considered in the above theorem, in the case of $\mathbb{P}^{1}$; see Kumar et al. ([8]) and Li ([9]). These authors use elements of $W_{l}\left(\mathbb{F}_{q}\right)[x]$, instead of $W_{l}\left(\mathbb{F}_{q}[x]\right)$, to form their exponential sums. The latter is a bigger ring but, for exponential sums, it doesn't matter. W. Li has informed us that their results can be deduced from the above theorem. In any rate, we can directly recover the applications in 8 to low-correlation sequences by following the same procedure of section 5 , replacing the multiplicative group by the elliptic curve. 


\section{CANONiCAL LifTingS}

Let $E$ be an ordinary elliptic curve defined over a finite field $\mathbb{F}_{q}$. Then $E$ has a canonical lifting to an elliptic curve over $W\left(\mathbb{F}_{q}\right)$ for which the Frobenius of $E$ also lifts. This is a special case of the Serre-Tate theory (see [11] or [7]). If $\mathbf{E}$ denotes the lift of $E$ to $W\left(\mathbb{F}_{q}\right)$, there is also an injective homomorphism $\tau: E\left(\overline{\mathbb{F}}_{q}\right) \rightarrow \mathbf{E}\left(W\left(\overline{\mathbb{F}}_{q}\right)\right)$ (analogous to the Teichmüller lift for $\mathbb{G}_{m}$ ), compatible with the action of Frobenius, which we will call the elliptic Teichmüller lift (see 2]). In fact, a characterization of the canonical lift is the existence of such a homomorphism. We will recall its construction below.

On the other hand, $\mathbf{E}$ has a Greenberg transform $G(\mathbf{E})$ which is an infinitedimensional scheme over $\mathbb{F}_{q}$, together with a map $\gamma: \mathbf{E}\left(W\left(\overline{\mathbb{F}}_{q}\right)\right) \rightarrow G(\mathbf{E})\left(\overline{\mathbb{F}}_{q}\right)$. Our purpose is to compute the degrees of the Witt coordinate functions of $\gamma \circ \tau$. By [2] we know that $\gamma \circ \tau$ actually corresponds to a section of the canonical morphism of

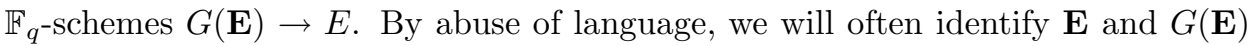
in what follows.

Theorem 4.1. Let $E$ be an ordinary elliptic curve defined over a finite field $\mathbb{F}_{q}$, and $\mathbf{E}$ the canonical lift of $E$ to $W\left(\mathbb{F}_{q}\right)$. Let $\mathbf{G}$ be an effective Cartier divisor on $\mathbf{E}$, and $G$ its restriction to $E$. Let $\mathbf{f}$ be a global section of $\mathcal{O}_{\mathbf{E}}(\mathbf{G})$. Then for $P \in E, P \notin \operatorname{supp} \mathbf{G}$, we have $\mathbf{f}(\tau(P))=\left(f_{0}(P), f_{1}(P), \ldots\right)$ as a Witt vector, where $f_{i}$ is a global section of $\mathcal{O}_{E}\left((2 p)^{i} G\right)$ for $i=0,1, \ldots$

Proof. A procedure for computing the $f_{i}$ 's is given in [3, Lemmas 2.6 and 2.7. First one computes the $p$-jet coordinates, $g_{i}$ say, which are reductions modulo $p$ of $\delta^{i} \mathbf{f}$, where $\delta u=\left(u \circ \phi-u^{p}\right) / p$ is a $p$-derivation on the structure sheaf of $\mathbf{E} / W\left(\mathbb{F}_{q}\right)$ and $\phi$ is the lift of Frobenius on $\mathbf{E} / W\left(\mathbb{F}_{q}\right)$, as follows from Lemma 2.7 of [3] together with [2]. As $\phi$ is an isogeny of degree $p$, it follows that $\operatorname{deg} \delta u$ is at most $2 p \operatorname{deg} u$ and therefore, by induction, $\operatorname{deg} g_{i} \leq(2 p)^{i} \operatorname{deg} G$. Now, from Lemma 2.6 of [3], we have $f_{i}+P_{i}\left(f_{0}, \ldots, f_{i-1}\right)=g_{i}$, for universal polynomials $P_{i}$ computed in the proof there. On the other hand, it is clear that the $f_{i}$ are regular away from $\operatorname{supp} G$, and thus so are the $g_{i}$. Using the proof of Lemma 2.6 of [3], one can show that $P_{n}\left(z f_{0}, z^{p} f_{1}, \ldots, z^{p^{n-1}} f_{n-1}\right)=z^{p^{n}} P_{n}\left(f_{0}, f_{1}, \ldots, f_{n-1}\right)$ for $z \in K$, so that monomials of $P_{n}\left(f_{0}, f_{1}, \ldots, f_{n-1}\right)$ are of the form $f_{0}^{i_{0}} f_{1}^{i_{1}} \cdots f_{n-1}^{i_{n-1}}$, with $i_{0}+$ $p i_{1}+\cdots+p^{n-1} i_{n-1}=p^{n}$. This implies that

$$
\operatorname{deg}\left(P_{n}\left(f_{0}, f_{1}, \ldots, f_{n-1}\right)\right) \leq \max \left\{p^{n-i} \operatorname{deg} f_{i} \mid 0 \leq i \leq n-1\right\} .
$$

A straightforward induction argument now gives $\operatorname{deg} f_{i} \leq(2 p)^{i} \operatorname{deg} G$, and the theorem then follows.

Suppose that $\mathbf{x}, \mathbf{y}$ are coordinates of a Weierstrass equation for $\mathbf{E}$. The above proof then produces functions $x_{0}, x_{1}, \ldots, y_{0}, y_{1}, \ldots$ on $E$ such that $x_{0}, y_{0}$ are the coordinates of the reduced Weierstrass equation for $E$ and

$$
\tau\left(\left(x_{0}, y_{0}\right)\right)=\left(\left(x_{0}, x_{1}, \ldots\right),\left(y_{0}, y_{1}, \ldots\right)\right) .
$$

By reducing modulo $p^{l}$, we can consider the canonical lift of $E$ to $W_{l}\left(\mathbb{F}_{q}\right)$. The following proposition gives us tools to help explicitly calculate this in the important case $l=2$, and is helpful when considering specific examples.

Proposition 4.2. Let $k$ be a perfect field of characteristic $p>0$, and $E / k$ an ordinary elliptic curve. If $\mathbf{E}$ is the canonical lift of $E$ to $W_{2}(k)$, then $\operatorname{deg} x_{1}<3 p$ and $\operatorname{deg} y_{1}<4 p$. Conversely, let $\mathbf{E}$ be any elliptic curve defined over $W_{2}(k)$ with 
reduction $E$. Assume that the projection given by reduction from $G(\mathbf{E})$ to $E$ admits a section $\tau$ in the category of $k$-schemes over $E \backslash\{O\}$ (where $O$ is the origin for the group law on $E)$ given by $\left(x_{0}, y_{0}\right) \mapsto(\mathbf{x}, \mathbf{y})=\left(\left(x_{0}, x_{1}\right),\left(y_{0}, y_{1}\right)\right)$, where $x_{1}, y_{1}$ are regular away from $O$ and satisfy $\operatorname{deg} x_{1}<3 p, \operatorname{deg} y_{1}<4 p$. Then $\tau$ is regular at $O, \mathbf{E}$ is the the canonical lift of $E$, and $\tau$ is the elliptic Teichmüller lift.

Proof. Theorem 4.1 gives that $\operatorname{deg} x_{1} \leq 4 p$, but since both $\mathbf{x} \circ \phi$ and $\mathbf{x}^{p}$ have a pole at the origin of $\mathbf{E}$, we actually get $\operatorname{deg} x_{1}<4 p$. Here again, $\phi$ denotes the lift of Frobenius on $\mathbf{E}$. Consider the differential $\phi^{*}(d \mathbf{x} / \mathbf{y}) / p$. As shown by Mazur 12], this is a well-defined, holomorphic differential on $\mathbf{E}$ and its reduction modulo $p, \omega$ say, depends only on $d x / y$. Moreover $C(\omega)=d x / y$, where $C$ is the Cartier operator. Hence $\omega=A^{-1} d x / y$, where $A$ is the Hasse invariant of $E$. On the other hand, from the proof of Theorem 4.1,

$$
\frac{1}{p} \phi^{*}\left(\frac{d \mathbf{x}}{\mathbf{y}}\right)=\frac{1}{p} \frac{d\left(\mathbf{x}^{p}+p x_{1}\right)}{\mathbf{y}^{p}+p y_{1}}=\frac{x_{0}^{p-1} d x_{0}+d x_{1}}{y_{0}^{p}} .
$$

This gives $d x_{1} / d x_{0}=A^{-1} y_{0}^{p-1}-x_{0}^{p-1}$, which is a polynomial in $x_{0}$ of degree $3(p-1) / 2$. Since $\operatorname{deg} x_{1}<4 p$, this determines $x_{1}$ up to a linear combination of 1 and $x_{0}^{p}$, and thus $x_{1}$ is a polynomial in $x_{0}$ of degree $(3 p-1) / 2$, hence $\operatorname{deg} x_{1}<3 p$. Examining the Weierstrass equation for $\mathbf{E}$ gives the bound for $y_{1}$.

To show the converse, first we need to show that $\tau$ is regular at $O$ and $\tau(O)=\mathbf{O}$, where $\mathbf{O}$ is the origin for the group law on $\mathbf{E}$. It is enough to show that $\mathbf{x} / \mathbf{y}$ is regular at $\mathbf{O}$ and that $\mathbf{x} / \mathbf{y}(\mathbf{O})=0$. A computation gives $\mathbf{x} / \mathbf{y}=\left(x_{0} / y_{0}, x_{1} / y_{0}^{p}-y_{1} x_{0}^{p} / y_{0}^{2 p}\right)$, and both $x_{1} / y_{0}^{p}$ and $y_{1} x_{0}^{p} / y_{0}^{2 p}$ vanish at $O$, since $\operatorname{deg} x_{1}<3 p, \operatorname{deg} y_{1}<4 p$.

Fix $P_{0} \in E, P_{0} \neq O$, and consider $f(P)=\tau\left(P+P_{0}\right)-\tau(P)-\tau\left(P_{0}\right)$. So $f$ is a morphism from $E$ to $\operatorname{ker}(G(\mathbf{E}) \rightarrow E)$. However $E$ is projective and $\operatorname{ker}(G(\mathbf{E}) \rightarrow E)$ is affine, so $f$ is constant. But $f(O)=\mathbf{O}$, so $f=\mathbf{O}$ and $\tau$ is a homomorphism. From the definition of the canonical lift, this forces $\mathbf{E}$ to be the canonical lift of $E$. Finally, if $\tau^{\prime}$ is the elliptic Teichmüller lift, then $\tau-\tau^{\prime}$ is a morphism from $E$ to $\operatorname{ker}(G(\mathbf{E}) \rightarrow E)$. And $\left(\tau-\tau^{\prime}\right)(O)=\mathbf{O}$, so by the same argument as above, $\tau=\tau^{\prime}$.

Remark 4.3. Applying Proposition 4.2, we can check the following examples of canonical lifts. If $\mathbf{E}$ is given by $\mathbf{y}^{2}+\mathbf{x y}=\mathbf{x}^{3}-\mathbf{x}^{2}-2 \mathbf{x}-1$ over $W\left(\overline{\mathbb{F}}_{2}\right)$, then it is the canonical lift of its reduction modulo two. A computation gives $x_{1}=1, y_{1}=$ $x_{0}^{2}\left(1+y_{0}\right)$. More generally, if $k$ is a field of characteristic 2 and $a \in k, a \neq 0$, consider the elliptic curve $E / k$ given by $y_{0}^{2}+x_{0} y_{0}=x_{0}^{3}+a$. Its canonical lift to $W_{2}(k)$ is $\mathbf{y}^{2}+\mathbf{x y}=\mathbf{x}^{3}+\left(a, a^{2}\right)$, and the elliptic Teichmüller lift is given by $\mathbf{x}=\left(x_{0}, a\right), \mathbf{y}=$ $\left(y_{0},\left(x_{0}^{2}+x_{0}\right) y_{0}+x_{0}^{3}+a x_{0}^{2}+a\right)$. The canonical lift to $W_{2}(k)$ of $y_{0}^{2}=x_{0}^{3}+x_{0}^{2}+a$ over a field $k$ of characteristic three is $\mathbf{y}^{2}=\mathbf{x}^{3}+\mathbf{x}^{2}+(a, 0)$, and the elliptic Teichmüller lift is $\mathbf{x}=\left(x_{0}, x_{0}^{4}+(1-a) x_{0}^{3}+a x_{0}-a^{2}\right), \mathbf{y}=\left(y_{0}, x_{0}^{2} y_{0}\right)$. For another example, $\mathbf{y}^{2}=\mathbf{x}^{3}+\mathbf{x}$ is the canonical lift of its special fiber in characteristic five, and the elliptic Teichmüller lift to $W_{2}$ is given by $\left(x_{0}, y_{0}\right) \mapsto(\mathbf{x}, \mathbf{y})=\left(\left(x_{0}, x_{1}\right),\left(y_{0}, y_{1}\right)\right)$, where $x_{1}=4 x_{0}^{7}+x_{0}^{3}, y_{1}=y_{0}\left(x_{0}^{8}+2 x_{0}^{6}+2 x_{0}^{4}+x_{0}^{2}+3\right)$. These examples show that the bounds in Proposition 4.2 on $\operatorname{deg} x_{1}$ and $\operatorname{deg} y_{1}$ cannot be any smaller.

Corollary 4.4. Notation as in the theorem. If $\mathbf{f} \circ \tau \neq F(\mathbf{g})-\mathbf{g}+\mathbf{c}$, for any $\mathbf{g} \in W_{l}(K), \mathbf{c} \in W_{l}\left(\mathbb{F}_{q}\right)$, then 


$$
\left|\sum_{P \in E_{0}\left(\mathbb{F}_{q}\right)} e^{2 \pi i T(\mathbf{f}(\tau(P))) / p^{l}}\right| \leq\left((2 p)^{l-1} \operatorname{deg} G+1\right) q^{1 / 2} .
$$

If we are in the special case $l=2$ and $\mathbf{G}=r \mathbf{O}$, the exponential sum above can be bounded by $(2 r+2) q^{1 / 2}$.

Proof. The first bound follows by combining Theorem 3.1 with Theorem 4.1 If $\mathbf{E} / W_{2}(k)$ is the lift of an elliptic curve $E / k$ given by a Weierstrass equation with coordinates $\mathbf{x}, \mathbf{y}$, then the global sections of $\mathcal{O}_{\mathbf{E}}(r \mathbf{O})$ are of the form $A+B \mathbf{y}$, where $A$ and $B$ are polynomials in $\mathbf{x}$ of degrees at most $[r / 2]$ and $[(r-3) / 2]$ respectively. It follows from the examples in Remark 4.3 that, in the situation of Theorem 4.1 in characteristic two, we have $\operatorname{deg} f_{1} \leq 2 r+1$, which improves slightly on the bound $4 r$ coming from Theorem 4.1 Correspondingly, we can improve the bound in the above corollary to $(2 r+2) q^{1 / 2}$.

Remark 4.5. It follows from [3], Propositions 1.7 and 1.8, that, if there is a section of the reduction map from a projective curve $\mathbf{X} / W\left(\mathbb{F}_{q}\right)$ to its special fibre $X / \mathbb{F}_{q}$, then $X$ is of genus zero or one. If the genus is zero, then the section exists. If the genus is one and $X$ is ordinary, the section exists if and only if $\mathbf{X}$ is the canonical lift of $X$; hence the restrictions in the above result. It is possible to obtain sections of reductions of affine curves over Witt vectors of finite length, but the degree of the sections grow much faster than that given by the above theorem, so the bounds are correspondingly worse.

\section{Applications}

We now return to the study of Euclidean weights of algebraic geometric codes defined using elliptic curves over Galois rings. In order to apply the results of sections 3 and 4 above, we make a few extra assumptions. Let $\mathbf{E}$ be an elliptic curve defined over the Galois ring $A=G R\left(p^{l}, m\right)=W_{l}\left(\mathbb{F}_{q}\right)$, where $q=p^{m}$. Let $E$ be the fiber of $\mathbf{E}$ over the closed point of Spec $A$, i.e., the reduction modulo $p$ of $\mathbf{E}$. Then $E$ is an elliptic curve over $\mathbb{F}_{q}$. We now make the additional assumptions that $E$ is ordinary and that $\mathbf{E}$ is the canonical lift of $E$ to $A$ (by which we mean the reduction modulo $p^{l}$ of the canonical lift of $E$ to $W\left(\mathbb{F}_{q}\right)$, as discussed in section 4.)

Let $Z_{0}$ be an $A$-point on $\mathbf{E}$ and let $P_{0}$ be the corresponding $\mathbb{F}_{q}$-rational point of $E$. Set $E_{0}=E \backslash\left\{P_{0}\right\}$. Let $\left\{P_{1}, \ldots, P_{n}\right\}=E_{0}\left(\mathbb{F}_{q}\right)$, and let $\mathcal{Z}=\left\{Z_{1}, \ldots, Z_{n}\right\}$, where $Z_{i}=\tau\left(P_{i}\right)$ for $i=1, \ldots, n$ and $\tau$ is the canonical lifting of points from section 4 . Let $r \geq 1$ and set $\mathcal{L}=\mathcal{O}_{\mathbf{E}}\left(r Z_{0}\right)$.

Theorem 5.1. Let $A, \mathbf{E}, \mathcal{Z}, \mathcal{L}$ be as above. Let $C=C_{A}(\mathbf{E}, \mathcal{Z}, \mathcal{L})$, and let $T$ : $A \rightarrow W_{l}\left(\mathbb{F}_{p}\right)=\mathbb{Z} / p^{l} \mathbb{Z}$ denote the absolute trace map. Then the minimum squared Euclidean weight of $T(C)$ satisfies

$$
w_{E}^{2}(T(C)) \geq \min \left\{2 n-\left((2 p)^{l-1} r+1\right) p^{\frac{m}{2}}, 4 n \frac{\pi^{2}}{p^{2 l}}\left(1+\frac{\pi^{2}}{3 p^{2 l}}\right)\right\} .
$$

Proof. Simply choose the group law for $\mathbf{E}$ so that $Z_{0}$ is the origin and hence $P_{0}$ is the origin for $E$. Then this theorem is a direct consequence of Corollary 4.4 Indeed, we need only to bound the Euclidean weight of the images of those $\mathbf{f}$ of the form $F(\mathbf{g})-\mathbf{g}+\mathbf{c}$, where $\mathbf{g} \in W_{l}(K), \mathbf{c} \in W_{l}\left(\mathbb{F}_{q}\right)$. In this case, $\left(T\left(\mathbf{f}\left(P_{1}\right)\right), \ldots, T\left(\mathbf{f}\left(P_{n}\right)\right)\right)$ is a multiple of the all-one vector, so the bound follows from the discussion in section 2 . 
In the case of elliptic curves over $\mathbb{Z} / 4 \mathbb{Z}$, we can refine the above result as follows.

Theorem 5.2. Use the same notation as above, but now assume that $p=l=2$, so that $T(C)$ is a code over $\mathbb{Z} / 4 \mathbb{Z}$. Then the minimum Lee weight of $T(C)$, and hence the minimum Hamming weight of $\varphi(T(C))$, where $\varphi$ is the Gray map described in the introduction, satisfies

$$
w_{L}(T(C))=w_{H}(\varphi(T(C))) \geq n-(2 r+2) 2^{\frac{m-1}{2}} .
$$

Proof. Repeat the proof of Theorem 5.1 above, this time using the second bound in Corollary 4.4. The result then follows since $w_{H}(\varphi(T(C)))=w_{L}(T(C))=$ $\frac{1}{2} w_{E}^{2}(T(C))$, as explained in section 2 .

Our methods can also be used to construct low-correlation sequences for use in CDMA (Code Division Multiple Access) communications systems, which are used in applications such as cellular telephones. For details on this, the reader is referred to $[8]$ and the references therein. We include here only a very brief overview of the basic idea. We consider infinite sequences of period $n$ with symbols in $\mathbb{Z} / p^{l} \mathbb{Z}$. The idea is to form a large family of such sequences which are pairwise cyclically distinct (i.e. no sequence is a shift of any other) and which have small correlation. Here the correlation $c_{s t}(\Delta)$ between sequences $s=\{s(j)\}$ and $t=\{t(j)\}$ of period $n$ for shift $\Delta$ is

$$
c_{s t}(\Delta)=\left|\sum_{j=0}^{n-1} e^{\frac{2 \pi i(s(j+\Delta)-t(j))}{p^{l}}}\right| .
$$

One measures whether or not a family of sequences is good by considering the maximum correlation parameter

$$
C_{\max }=\max \left\{c_{s t}(\Delta) \mid s, t \in \mathcal{F} \text { and either } s \neq t \text { or } \Delta \neq 0\right\} .
$$

In applications, the large family size allows for a large number of users, and a small correlation parameter translates to little interference from one user to another.

In order to describe how to construct a family of sequences from an elliptic curve, we will need the following lemma.

Lemma 5.3. Let $E / \mathbb{F}_{q}$ be an elliptic curve with $n$ rational points and let $r>1$ be an integer, $(r, n)=1$. For any point $Q \in E\left(\mathbb{F}_{q^{r}}\right)$ and for any $\sigma$ in the Galois group of $\mathbb{F}_{q}(Q) / \mathbb{F}_{q}$, we have that $Q^{\sigma}-Q \notin E\left(\mathbb{F}_{q}\right) \backslash\{O\}$.

Proof. Let $\sigma$ be of order $d \mid r$. If $Q^{\sigma}-Q=P_{0} \in E\left(\mathbb{F}_{q}\right)$, then

$$
O=Q^{\sigma^{d}}-Q=\sum_{i=1}^{d} Q^{\sigma^{i}}-Q^{\sigma^{i-1}}=\sum_{i=1}^{d}\left(Q^{\sigma}-Q\right)^{\sigma^{i-1}}=d P_{0} .
$$

However, since $d$ is coprime to $n$, this implies $P_{0}=O$, proving the lemma.

Now we describe how to construct a family of sequences. Take $\mathbf{E}$ as above. Assume that $E\left(\mathbb{F}_{q}\right)$ is cyclic of order $n$, and let $P_{1}$ be its generator. Let $Q$ be as in Lemma 5.3. Each point in the Galois orbit of $Q$ lifts to an element of $\mathbf{E}\left(W_{l}\left(\mathbb{F}_{q^{r}}\right)\right)$. Let $\mathbf{G}$ be the Cartier divisor on $\mathbf{E}$ which is the sum of these lifts, and let $G$ be the reduction of $\mathbf{G}$. In particular $\mathbf{G}$ is a sum of distinct points of $W_{l}\left(\mathbb{F}_{q^{r}}\right)$, but because these distinct points form a Galois orbit, $\mathbf{G}$ exists as a divisor over $W_{l}\left(\mathbb{F}_{q}\right)$. Also, $G$ is simply the divisor of degree $r$ on $E / \mathbb{F}_{q}$ corresponding to the Galois orbit of $Q$. For each equivalence class in $\Gamma\left(\mathbf{E}, \mathcal{O}_{\mathbf{E}}(\mathbf{G})\right) / W_{l}\left(\mathbb{F}_{q}\right)$, choose a representative $\mathbf{f}$ in 
$\Gamma\left(\mathbf{E}, \mathcal{O}_{\mathbf{E}}(\mathbf{G})\right)$ and define the sequence $s_{\mathbf{f}}(j)=T\left(\mathbf{f}\left(\tau\left(j P_{1}\right)\right)\right)$. The set $\mathcal{F}$ of all such sequences is our family.

Theorem 5.4. Let $\mathcal{F}$ be the family of sequences defined above. Then the maximum correlation parameter $C_{\max }$ of $(\mathcal{F})$ satisfies

$$
C_{\max } \leq 1+\left((2 p)^{l-1} 2 \operatorname{deg} \mathbf{G}+1\right) p^{\frac{m}{2}} .
$$

Furthermore, if $\operatorname{deg} \mathbf{G}$ and the above bound for $C_{\max }$ are both less than $n$, then $|\mathcal{F}|=q^{l(\operatorname{deg} \mathbf{G}-1)}$.

Proof. The correlation of $s_{\mathbf{f}}$ and $s_{\mathbf{g}}$ for shift $\Delta$ is

$$
\left|\sum_{j=1}^{n} e^{2 \pi i T\left(\mathbf{f}\left(\tau\left(j P_{1}\right)\right)-\mathbf{g}\left(\tau\left((j+\Delta) P_{1}\right)\right)\right) / p^{l}}\right| .
$$

Consider the automorphism $\alpha$ of $\mathbf{E}$ given by translation by $\tau\left(\Delta P_{1}\right)$. Let $\mathbf{h}=\mathbf{f}-$ go $\alpha$. The above sum is then the kind of sum considered in Corollary 4.4 say, but with $\mathbf{G}$ replaced by $\mathbf{G}+\alpha^{*} \mathbf{G}$. If $\mathbf{h} \circ \tau$ is not of the form $F(\mathbf{k})-\mathbf{k}+\mathbf{c}$, where $\mathbf{k} \in W_{l}(K)$ and $\mathbf{c} \in W_{l}\left(\mathbb{F}_{q}\right)$, then the result follows from Corollary 4.4 since the degree of the divisor $\mathbf{G}+\alpha^{*} \mathbf{G}$ is at most $2 \operatorname{deg} \mathbf{G}$.

We now assume that $\mathbf{h}$ is of the type excluded by Corollary 4.4, so that $\mathbf{h} \circ \tau=$ $F(\mathbf{k})-\mathbf{k}+\mathbf{c}$ for some $\mathbf{k} \in W_{l}(K)$ and $\mathbf{c} \in W_{l}\left(\mathbb{F}_{q}\right)$. The first Witt coordinate of the equation $(\mathbf{f}-\mathbf{g} \circ \alpha) \circ \tau=F(\mathbf{k})-\mathbf{k}+\mathbf{c}$ is an equation of the form $f_{0}-g_{0} \circ \alpha=$ $k_{0}^{p}-k_{0}+c_{0}$. Now from our hypothesis $f_{0}$ and $g_{0}$ have simple poles, so $f_{0}-g_{0} \circ \alpha$ also has simple poles. But $k_{0}^{p}-k_{0}+c_{0}$ won't have simple poles unless it is constant, so $f_{0}-g_{0} \circ \alpha$ is constant.

If $\Delta \neq 0$, Lemma 5.3 ensures that $G$ and $\alpha^{*} G$ have disjoint support, and therefore $f_{0}$ and $g_{0} \circ \alpha$ have disjoint polar divisors unless $f_{0}$ and $g_{0}$ are both constants. Therefore, $f_{0}-g_{0} \circ \alpha$ constant implies that $f_{0}$ and $g_{0}$ are both constants. We take the (usual) Teichmüller lifts of these constants and subtract them from $\mathbf{f}, \mathbf{g}$, so that we may assume that $f_{0}, g_{0}$ are both zero. This implies that $\mathbf{f}=p \mathbf{f}^{\prime}$ and $\mathbf{g}=p \mathbf{g}^{\prime}$ for some $\mathbf{f}^{\prime}, \mathbf{g}^{\prime} \in \mathbf{K}$, where $\mathbf{K}$ is the total quotient ring of rational functions on $\mathbf{E}$. Using Lemma 2.4, we can write $\mathbf{f}=p \mathbf{f}^{\prime}, \mathbf{g}=p \mathbf{g}^{\prime}$, where $\mathbf{f}^{\prime}$ and $\mathbf{g}^{\prime}$ are in the space of global sections of the line bundle associated to $\mathbf{G}$. As in the proof of Theorem 3.1, we may consider $\mathbf{f}^{\prime}$ and $\mathbf{g}^{\prime}$ to be defined over $W_{l-1}(K)$, so we use induction. Either we have a bound for the exponential sum formed with $\mathbf{f}^{\prime}-\mathbf{g}^{\prime} \circ \alpha$ which is better than the bound in the statement of the theorem, or we can repeat the process and finally get that $\mathbf{f}$ and $\mathbf{g}$ are constant, and this possibility is excluded by the construction of our family.

If $\Delta=0$, then $\alpha$ is the identity, so $f_{0}-g_{0}=c_{0}$, a constant. In order to compute correlations, the choice of the representative $\mathbf{f}$ is immaterial, so we may subtract from $\mathbf{f}$ the (usual) Teichmüller lift of $c_{0}$ and assume that $c_{0}=0$. As above, $\mathbf{f}-\mathbf{g}=p \mathbf{h}$, for some $\mathbf{h}$ in $\Gamma\left(\mathbf{E}, \mathcal{O}_{\mathbf{E}}(\mathbf{G})\right)$, and we can proceed by induction on $l$ as before.

To estimate $|\mathcal{F}|$, we note that $s_{\mathbf{f}}=s_{\mathbf{g}}$ implies that the correlation of the two sequences is $n$, so by the above argument $\mathbf{f}-\mathbf{g}$ is a constant; hence $|\mathcal{F}|=$ $\left|\Gamma\left(\mathbf{E}, \mathcal{O}_{\mathbf{E}}(\mathbf{G})\right)\right| /\left|W_{l}\left(\mathbb{F}_{q}\right)\right|$. Finally, $\Gamma\left(\mathbf{E}, \mathcal{O}_{\mathbf{E}}(\mathbf{G})\right)$ is a free $W_{l}\left(\mathbb{F}_{q}\right)$-module of rank $\operatorname{deg} \mathbf{G}$, by Theorem 2.2 . 


\section{EXAMPLES AND DISCUSSION}

In order to construct a $\mathbb{Z} / 4 \mathbb{Z}$-code, or, more specifically, a binary code, using the methods described in this paper, one would begin with an elliptic curve defined over the ring $W_{2}\left(\mathbb{F}_{2^{m}}\right)$ of length 2 Witt vectors over a finite field of characteristic 2. From this curve, one would construct an algebraic geometric code over the ring $W_{2}\left(\mathbb{F}_{2^{m}}\right)$. Applying the trace map, one obtains a code over the ring $\mathbb{Z} / 4 \mathbb{Z}$, and then applying the Gray map yields the binary code. For sufficiently large $m$, virtually any elliptic curve should yield a good code. Further, we believe that good examples can be constructed using smaller fields, but our bounds do not ensure that this is the case. In particular, our bounds are not useful for parameters within the range of current tables of codes 1, 10. We did some brute force calculations to compute these parameters for some codes, but an exhaustive search proved prohibitive and we did not find a code beating the known bounds on the tables. However, we offer the following two examples as a partial demonstration of the potential of these methods.

6.1. First example. Let $\mathbf{E}$ be the curve over $W_{2}\left(\overline{\mathbb{F}}_{2}\right)$ defined by the equation $\mathbf{y}^{2}+\mathbf{x y}=\mathbf{x}^{3}-\mathbf{x}^{2}-2 \mathbf{x}-1$, so that its reduction modulo 2 is the curve $E$ over $\overline{\mathbb{F}}_{2}$ defined by the equation $y^{2}+x y=x^{3}+x^{2}+1$. (Although $E$ is isomorphic over $\overline{\mathbb{F}}_{2}$ to the curve with equation $y^{2}+x y=x^{3}+1$, we work with $E$ rather than this latter curve because $E$ has more $\mathbb{F}_{8}$-rational points.) It is easy to check that if $\left(x_{0}, y_{0}\right)$ is a point on $E$, then $\left(\left(x_{0}, 1\right),\left(y_{0}, x_{0}^{2}\left(1+y_{0}\right)\right)\right)$ is a point on E. Further, the map $\tau: E\left(\overline{\mathbb{F}}_{2}\right) \rightarrow \mathbf{E}\left(W_{2}\left(\overline{\mathbb{F}}_{2}\right)\right)$ given by $\left(x_{0}, y_{0}\right) \mapsto\left(\left(x_{0}, 1\right),\left(y_{0}, x_{0}^{2}\left(1+y_{0}\right)\right)\right)$ satisfies the hypotheses of Proposition 4.2 above, so we can conclude that $\mathbf{E}$ is the canonical lift of $E$. We will construct a code over $W_{2}\left(\mathbb{F}_{8}\right)$ using $\mathbf{E}$. Applying the trace map will give us a code over $\mathbb{Z} / 4 \mathbb{Z}$, and applying the Gray map will give us a binary code.

Write $\mathbb{F}_{8}=\mathbb{F}_{2}[t] /\left(t^{3}+t+1\right)$. In addition to the origin $O$, there are 13 finite $\mathbb{F}_{8}$-rational points on $E$. Applying the map $\tau$ described above, one gets the origin $\mathbf{O}$ of $\mathbf{E}$ and thirteen points of $\mathbf{E}$ defined over $W_{2}\left(\mathbb{F}_{8}\right)$. Let $\mathcal{Z}$ be the set containing these thirteen points. We wish to consider the code $C=C_{W_{2}\left(\mathbb{F}_{8}\right)}(\mathbf{E}, \mathcal{Z}, \mathcal{L})$, where $\mathcal{L}=\mathcal{O}_{\mathbf{E}}(3 \mathbf{O})$. The degree of $\mathcal{L}$ is 3 , so by the Riemann-Roch Theorem [20, the rank of the free $W_{2}\left(\mathbb{F}_{8}\right)$-module $\Gamma(\mathbf{E}, \mathcal{L})$ is 3 . It is easy to check that $\{1, \mathbf{x}, \mathbf{y}\}$ is a basis for this module, so a $(3 \times 13)$ generator matrix for $C$ is constructed simply by evaluating these three functions at each of the thirteen points in $\mathcal{Z}$.

In fact, we are interested in the trace code $T(C)$ of $C$, which will be a code over $W_{2}\left(\mathbb{F}_{2}\right)=\mathbb{Z} / 4 \mathbb{Z}$, and the $(13 \times 7)$ matrix obtained by evaluating the functions 1 , $\mathbf{x}, t \mathbf{x}, t^{2} \mathbf{x}, \mathbf{y}, t \mathbf{y}$, and $t^{2} \mathbf{y}$ has rank 7 and is a generator matrix for our trace code.

Unfortunately, Theorem 5.2 only tells us that the minimum Lee weight of this code is at least $13-(2(3)+2) 2^{\frac{3-1}{2}}=-3$, so it's not very useful. However, a brute

force check (using Mathematica or Pari) shows that the minimum Lee weight of this code is 5. Further, by appending a check digit which is the sum of the first three coordinates, we get a code over $\mathbb{Z} / 4 \mathbb{Z}$ of length 14 , rank 7 , and minimum Lee weight 6 . The generator matrix is

$$
\left(\begin{array}{llllllllllllll}
3 & 3 & 3 & 3 & 3 & 3 & 3 & 3 & 3 & 3 & 3 & 3 & 3 & 1 \\
2 & 0 & 0 & 0 & 0 & 0 & 0 & 3 & 3 & 3 & 3 & 3 & 3 & 2 \\
0 & 1 & 1 & 2 & 2 & 1 & 1 & 3 & 3 & 2 & 2 & 1 & 1 & 2 \\
0 & 2 & 2 & 1 & 1 & 1 & 1 & 2 & 2 & 1 & 1 & 3 & 3 & 0 \\
3 & 3 & 1 & 1 & 3 & 3 & 1 & 2 & 3 & 2 & 3 & 2 & 3 & 3 \\
2 & 0 & 3 & 1 & 1 & 1 & 2 & 2 & 3 & 0 & 2 & 2 & 1 & 1 \\
2 & 1 & 1 & 2 & 1 & 0 & 3 & 0 & 2 & 2 & 1 & 2 & 3 & 0
\end{array}\right) .
$$


Applying the Gray map gives a binary code of length 28 with $2^{14}=16384$ codewords and minimum Hamming distance 6 . The best code with this length and number of codewords has minimum Hamming distance 8.

It is interesting to note that the first four rows and the odd-numbered columns of the generator matrix above define the $[7,4]$ Hamming code over $\mathbb{Z} / 4 \mathbb{Z}$ (see [6]). Since only the functions 1 and $\mathbf{x}$ are used here, this gives a construction of this code as a trace code of an algebraic geometric code over $\mathbb{P}^{1}$. For a more direct construction of the $[7,4]$ Hamming code over $\mathbb{Z} / 4 \mathbb{Z}$ as an algebraic geometric code, see [21]. For more details on the above example, see [18].

6.2. Second example. For this example, consider the curve $E$ given by the equation $y^{2}+y=x^{3}+t^{3}$ over the field $\mathbb{F}_{16}:=\mathbb{F}_{2}[t] /\left(t^{4}+t+1\right)$. This curve is supersingular, so we cannot consider its canonical lift. However, we develop the theory of lifting points on affine plane curves when no canonical lift exists in another paper [19]. In this case it is easy to see that the curve $\mathbf{E}$ over $W_{2}\left(\mathbb{F}_{16}\right)$ given by the equation $\mathbf{y}^{2}+\mathbf{y}=\mathbf{x}^{3}+\left(t^{3}, 0\right)$ certainly has $E$ as its reduction. Further, it is easy to check that whenever $\left(x_{0}, y_{0}\right)$ is an affine point on $E, \lambda\left(\left(x_{0}, y_{0}\right)\right):=\left(\left(x_{0}, 0\right),\left(y_{0}, y_{0}^{3}+x_{0}^{3} t^{3}\right)\right)$ satisfies the equation defining $\mathbf{E}$.

The curve $E$ has 24 affine $\mathbb{F}_{16}$-rational points. If we follow the method of the previous example, using the basis $\{1, \mathbf{x}, \mathbf{y}\}$ for the global sections of $\mathcal{O}_{\mathbf{X}}(3 \mathbf{O})$ on $\mathbf{E}$, we get a binary code of length 48 with $2^{18}$ codewords and minimum distance 8 . As the best linear code of this length with this many codewords has minimum distance somewhere between 12 and 14, this is not a good code.

However, if we evaluate the rational functions in $\Gamma\left(\mathbf{X}, \mathcal{O}_{\mathbf{X}}(2 \mathbf{O})\right)$ (using the basis $\{1, \mathbf{x}\}$ ) at the lifts of only half the points, we get a pretty good code. In particular, it is easy to see that the affine $F_{16}$-rational points on $E$ occur in pairs sharing the same $x$-coordinate. Taking one point from each of these pairs, lifting them and evaluating the functions 1 and $\mathbf{x}$ at these lifts yields a code whose trace code has generator matrix

$$
\left(\begin{array}{llllllllllll}
3 & 3 & 3 & 3 & 3 & 3 & 3 & 3 & 3 & 3 & 3 & 3 \\
1 & 3 & 2 & 3 & 2 & 3 & 3 & 1 & 2 & 1 & 2 & 1 \\
1 & 0 & 2 & 1 & 3 & 0 & 3 & 2 & 0 & 1 & 3 & 0 \\
3 & 1 & 1 & 2 & 0 & 2 & 3 & 3 & 1 & 2 & 2 & 0 \\
2 & 0 & 3 & 2 & 3 & 2 & 3 & 1 & 2 & 1 & 0 & 1
\end{array}\right)
$$

The image under the Gray mapping of this code is a binary code of length 24 with $2^{10}$ codewords and minimum Hamming distance 8 . This matches the best possible binary linear code with this length and number of codewords.

\section{ACKNOWLEDGMENTS}

The authors acknowledge the use of the software packages Mathematica and Pari in some calculations. The first author would also like to thank A. Buium and J. Tate for useful comments. The second author thanks Winnie Li and Patrick Solé for their useful comments.

\section{REFERENCES}

1. A. E. Brouwer and T. Verhoeff, An updated table of minimum-distance bounds for binary linear codes, IEEE Trans. Inform. Theory 39 (1993), 662-677, supplemented by on-line updates: information regarding $[n, k, d]$ codes with fixed $n, k$ is accessible over the World Wide Web via http://www.win.tue.nl/win/math/dw/voorlincod.html. MR 94d:94010 
2. A. Buium, An approximation property for Teichmüller points, Math. Research Letters 3 (1996), 453-457. MR 97m:14026

3. L Geometry of p-jets, Duke Math. Journal 82 (1996), 349-367. MR 97c:14029

4. P. Deligne, Applications de la formule des traces aux sommes trigonométriques, Cohomologie étale (SGA 4 $\frac{1}{2}$ ), Lecture Notes in Math, vol. 569, Springer-Verlag, Berlin, Heidelberg, New York, 1977. MR 57:3132

5. V. D. Goppa, Codes associated with divisors, Probl. Peredachi Inf. 13 (1977), 33-39, English translation in Probl. Inf. Transm., 13:22-27, (1977). MR 58:15672

6. A. R. Hammons Jr., P. V. Kumar, A. R. Calderbank, N. J. A. Sloane, and P. Solé, The $\mathbb{Z}_{4}$-linearity of Kerdock, Preparata, Goethals, and related codes, IEEE Transactions on Information Theory 40 (1994), 301-319. MR 95k:94030

7. N. M. Katz, Serre-Tate local moduli, Algebraic surfaces (Orsay, 1976-78), Lecture Notes in Math., vol. 868, Springer, Berlin-New York, 1981, pp. 138-202. MR 83k:14039b

8. P. V. Kumar, T. Helleseth, and A. R. Calderbank, An upper bound for Weil exponential sums over Galois rings and applications, IEEE Transactions on Information Theory 41 (1995), 456-468. MR 96c:11140

9. W-C. W. Li, Character sums over p-adic fields, J. Number Theory 74 (1999), 181-229. CMP 99:08

10. S. Litsyn, E. M Rains, and N. J. A. Sloane, Table of nonlinear binary codes, available on the World Wide Web at http://www.research.att.com/ njas/codes/And/.

11. J. Lubin, J-P. Serre, and J. Tate, Elliptic curves and formal groups, Proc. of the Woods Hole summer institute in algebraic geometry, 1964.

12. B. Mazur, Frobenius and the Hodge filtration, estimates, Ann. Math. 98 (1973), 58-95. MR 48:297

13. H. L. Schmid, Zur arithmetik der zyklischen p-Körper, Crelles J. 176 (1936), 161-167.

14. K_ Kongruenzzetafunktionen in zyklischen Körpern, Abh. Preuss. Akad. Wiss. Math.Nat. Kl. 1941 (1942), 30pp. MR 8:310b

15. H. Stichtenoth, Algebraic function fields and codes, Springer, Berlin, 1993. MR 94k:14016

16. M. A. Tsfasman and S. G. Vlăduţ, Algebraic-geometric codes, Kluwer, Dordrecht, 1991. MR 93i:94023

17. M. A. Tsfasman, S. G. Vlăduţ, and Th. Zink, Modular curves, Shimura curves, and Goppa codes, better than the Varshamov-Gilbert bound, Math. Nachrichten 109 (1982), 21-28. MR 85i: 11108

18. J.-F. Voloch and J. L. Walker, Lee weights of Z/4Z-codes from elliptic curves, to appear in Codes, Curves, and Signals: Common Threads in Communications.

19. 1768-1776. CMP 2000:03

20. J. L. Walker, Algebraic geometric codes over rings, to appear in the Journal of Pure and Applied Algebra 144 (1999), 91-110. CMP 2000:04

21. - The Nordstrom Robinson code is algebraic geometric, IEEE Transactions on Information Theory 43 (1997), 1588-1593. MR 98k:94014

Department of Mathematics, University of Texas, Austin, Texas 78712

E-mail address: voloch@math.utexas.edu

Department of Mathematics and Statistics, University of Nebraska, Lincoln, NeBRASKA 68588-0323

E-mail address: jwalker@math.unl.edu 